\title{
Unveiling a Selective Mechanism for the Inhibition of $\alpha$-Synuclein Aggregation by $\beta$-Synuclein
}

\author{
Andre Leitao 1,2, Akshay Bhumkar 1,2, Dominic J. B. Hunter 1,3, Yann Gambin 1,2,* \\ and Emma Sierecki 1,2,* \\ 1 European Molecular Biology Laboratory (EMBL), Australia Node in Single Molecule Science, \\ Sydney NSW 2031, Australia; a.leitao@unsw.edu.au (A.L.); a.bhumkar@unsw.edu.au (A.B.); \\ d.hunter@imb.uq.edu.au (D.J.B.H.) \\ 2 School of Medical Sciences, The University of New South Wales, Sydney NSW 2031, Australia \\ 3 Institute for Molecular Bioscience, The University of Queensland, St Lucia QLD 4076, Australia \\ * Correspondence: y.gambin@unsw.edu.au (Y.G.); e.sierecki@unsw.edu.au (E.S.); Tel.: +61-(2)-9385-8933 (Y.G. \& E.S.)
}

Received: 8 December 2017; Accepted: 19 January 2018; Published: 24 January 2018

\begin{abstract}
Synuclein $(\alpha S)$ is an intrinsically disordered protein that is associated with Parkinson's disease (PD) through its ability to self-assemble into oligomers and fibrils. Inhibition of this oligomerization cascade is an interesting approach to developing therapeutical strategies and $\beta$-synuclein ( $\beta S$ ) has been described as a natural negative regulator of this process. However, the biological background and molecular mechanisms by which this inhibition occurs is unclear. Herein, we focused on assessing the effect of $\beta S$ on the aggregation of five $\alpha S$ pathological mutants linked to early-onset PD (A30P, E46K, H50Q, G51D and A53T). By coupling single molecule fluorescence spectroscopy to a cell-free protein expression system, we validated the ability of $\beta S$ to act as a chaperone of $\alpha \mathrm{S}$, effectively inhibiting its aggregation. Interestingly, we found that $\beta S$ does so in a selective manner, i.e., is a more effective inhibitor for certain $\alpha \mathrm{S}$ pathological mutants-A30P and G51D—as compared to E46K, H50Q and A53T. Moreover, two-color coincidence experiments proved that this discrepancy is due to a preferential incorporation of $\beta S$ into smaller oligomers of $\alpha \mathrm{S}$. This was validated by showing that the chaperoning effect was lost when proteins were mixed after being expressed individually. This study highlights the potential of fluorescence spectroscopy to deconstruct $\alpha \mathrm{S}$ aggregation cascade and its interplay with $\beta S$.
\end{abstract}

Keywords: $\alpha$-synuclein; $\beta$-synuclein; Parkinson's disease; protein oligomerization; single molecule spectroscopy; number and brightness analysis; two-color coincidence

\section{Introduction}

Pathological protein aggregation is a poorly understood phenomenon that lies at the root of several neurodegenerative diseases including Parkinson's, Alzheimer's, Huntington's and Creutzfeld-Jakob diseases. For each of these diseases, one or more proteins have been shown to self-assemble into highly-ordered fibrils with further accumulation into amyloid deposits. In Parkinson's disease (PD), these intracellular deposits are called Lewy Bodies and are primarily composed of the presynaptic protein $\alpha$-Synuclein $(\alpha S)$ [1].

$\alpha$-Synuclein $(\alpha S)$ is amongst some of the most studied aggregation-prone proteins in neurodegeneration, mostly due to its involvement in the pathogenesis of Parkinson's disease (PD) and other disorders such as Dementia with Lewy Bodies (DLB) or Multiple Systems Atrophy (MSA), all of which are thus generically designated as synucleinopathies [2]. $\alpha \mathrm{S}$ is a small 140 amino-acid long protein, encoded by the gene SNCA, that predominantly localizes at the presynaptic terminals of neurons, where it comprises $1 \%$ of all cytosolic proteins. Although its exact biological function at the presynapse has not been fully elucidated, $\alpha \mathrm{S}$ is often associated with or in close proximity 
of synaptic vesicles, playing an important role in the trafficking of these vesicles, and in the regulation of neurotransmitter exocytosis through membrane remodelling [3,4].

The lack of a defined structured of $\alpha S^{\prime} s$ monomeric form in solution categorizes it as an intrinsically disordered protein (IDP) and partly explains the challenges associated with understanding its function and aggregation propensity in neurons [5]. In fact, $\alpha S^{\prime}$ s ability to self-assemble in PD is often depicted as an irreversible cascade of events that leads to the formation of different soluble oligomeric/protofibrillar species and culminates in the formation of insoluble fibrils that ultimately accumulate in Lewy Bodies [1]. Straightforward as this process may seem, it raises a multitude of questions. The succession and roles of each aggregated species are largely unknown and whether different oligomers are formed on- or off-pathway is still controversial. Furthermore, recent studies have shown intermediate oligomeric states to be more toxic than the mature fibrils $[6,7]$, challenging conventional views that have historically associated fibrils as the source of toxicity to neurons. However, whether this mechanism of toxicity is related to gain- or loss-of-function properties of $\alpha \mathrm{S}$ aggregates is unclear.

$\alpha S^{\prime}$ s aminoacid sequence provides us with some clues to its function and aggregation behaviour. The N-terminal part of the polypetide (residues 1-60) is characterized by the presence of six imperfect KTKEGV aminoacid repeats that confer a variation of hydrophobicity with a strict periodicity of 11 residues, a feature that is typical of amphipathic helices in apolipoproteins and critical for lipid or membrane binding $[3,5]$. The core of the molecule is designated the non- $\mathrm{A} \beta$ component (NAC) because it was first reported to be present in amyloid- $\beta$ deposits in patients with Alzheimer's disease. It encompasses residues 61-90 and is a highly hydrophobic region that shows high propensity for $\beta$-sheet formation, typical of later stages of fibrillation, as opposed to the acidic C-terminal region, which has been shown to be highly soluble [8]. Interconversion between oligomeric forms, which have been shown to be more flexible and characterized by antiparallel $\beta$-sheet, and fibrils with a parallel $\beta$-sheet core, has been postulated by several authors to be a key step in the progression of aggregation $[7,9]$. Furthermore, recent data indicate that oligomeric $\alpha$-synuclein species can spread between cells, and thereby act as seeds, propagating $\alpha \mathrm{S}$ pathology [10-13].

An additional factor that has hindered the understanding of $\alpha S^{\prime}$ s aggregation is the slow rate and very specific conditions (temperature, $\mathrm{pH}$, lipid content, etc.) at which it occurs. As it happens, five naturally-occurring single-point mutations in the SNCA gene have been reported to enhance $\alpha S^{\prime}$ s oligomerization in vitro and in vivo. In fact, although PD is predominantly sporadic in nature, $5-10 \%$ of cases are believed to be familial, leading to earlier-onset forms of the disease. Interestingly, all five different $\alpha \mathrm{S}$ missense mutations identified to date in familial forms of PD are contained within the N-terminal domain, and include: A30P, E46K, H50Q, G51D and A53T. Several studies have focused on the aggregation behaviour of these mutant forms of $\alpha \mathrm{S}$ and established a much higher propensity to form fibrils as compared to the WT $\alpha \mathrm{S}$ [14-17], attesting the importance of the N-terminal domain in the pathophysiology of $\mathrm{PD}$, particularly in earlier stages of oligomerization. Moreover, striking differences have been identified between the populations of aggregates formed by the different point-mutants regarding $\beta$-sheet content, conformational flexibility, intra- and intermolecular interactions and ability to permealize membranes [15,16,18-21].

Recent studies performed our group [22] using single molecule spectroscopy and a cell free protein expression system have painted a different picture of $\alpha S$ aggregation, where the formation of different aggregate species ultimately defines one of two possible pathways towards fibril formation: E46K, H50Q and A53T rapidly form large fibrils whereas A30P, G51D and WT aggregate less and form smaller objects. Moreover, these groups were shown to be mutually exclusive in their ability to recruit one another's aggregates.

Finding natural inhibitors of protein aggregation is crucial to both the fundamental understanding of the aggregation process and the development of future therapeutic strategies. Coincidently, $\alpha S$ belongs to a highly conserved family of two other proteins, $\beta$ - and $\gamma$-synuclein, both of which have been reported to inhibit $\alpha \mathrm{S}$ fibril formation [23]. However, whilst $\gamma$-synuclein is predominantly 
expressed in sensory- and motor-neurons of the peripheral nervous system and has not been implicated in synucleinopathies, $\beta$-synuclein ( $\beta S$ ) colocalizes with $\alpha S$ at the presynapse of CNS neurons, where both proteins are expressed at similar levels [24]. $\beta S$ has been observed to inhibit $\alpha S^{\prime}$ s aggregation both in vitro [23,25] and in vivo [26,27], raising interest in its potential role as a natural anti-parkinsonian agent. $\beta$ - and $\alpha$ S show a high degree of sequence homology $(61.6 \%)$, similar intrinsically disordered nature, as well as ability for lipid binding. Importantly, $\beta S$ lacks a 11 residue stretch at the NAC domain, which has been hypothesised as being the main cause for its lower propensity to form amyloid fibrils [23]. Up- and down-regulation of $\alpha S$ and $\beta S$, respectively, have been correlated with disease onset suggesting that altered relative expression levels of these proteins changes disease progression [28]. Furthermore, $\alpha-\beta S$ bigenic mice revealed amelioration of neurodegenerative effects compared with single $\alpha$ S transgenic mice [26]. Other in vivo evidence pointing to a neuroprotective role of $\beta S$ includes the utilization of $\beta S$-derived peptides as potential therapeutical strategies revealing phenotype recovery in mice [29] and fly models [30], or the intracerebral injection of lenti- $\beta S$ virus with subsequent reduction in the formation of $\alpha S$ plaques in mice [31]. Although it is now well established that $\beta S$ is able to modulate $\alpha S^{\prime} s$ aggregation, the mechanisms behind this phenomenon and its exact biological relevance are still under intense scrutiny.

The molecular mechanisms governing the regulatory effect between $\alpha \mathrm{S}$ and $\beta S$ inhibition have also been a source of interesting results, albeit somewhat more divergent: Tsigelny and co-workers were able to create cell-free dimeric and pore-like oligomeric forms of $\alpha \mathrm{S}$ and disrupt them with $\beta S$ [32]; other authors reveal the suppression of initiation and elongation of $\alpha \mathrm{S}$ aggregates via competitive binding to surfaces [33]. Similarly in cells, some studies point to the ability of $\beta S$ to prevent aggregated $\alpha \mathrm{S}$ from inhibiting the proteasome, while others suggest that the mechanism involves the reduction in $\alpha$ S expression by increased levels of $\beta S$ [34]. Recently, two missense mutations of $\beta S$ have been identified in unrelated cases of DLB-V70M and P123H [35]. These mutations have been shown to promote $\beta S$ aggregation and accumulation into lysosomal inclusion bodies [36]. Given the regulatory effect that $\beta S$ exerts on $\alpha S$ aggregation, these mutations could provide valuable information on the interplay between both proteins.

Here, we investigated the effect of $\beta S$ on different aggregated species of $\alpha S$, in order to understand which mechanisms define the interplay between these two highly homologous proteins. We developed an experimental method to efficiently coexpress these proteins in a cell-free system, bypassing delicate steps of protein purification and labelling that often irreversibly affect (co)aggregation behaviours, especially in the case of intrinsically disordered proteins. By using single molecule fluorescence techniques, our experimental setup proved robust enough to provide us with valuable insights into the relationship between synucleins.

\section{Results}

\subsection{Cell-Free Coexpression of $\alpha S$ and $\beta S$ Allows for Investigation of Their Inter-Regulatory Effect}

We first wanted to investigate whether we could design a robust coexpression setup with our cell-free protein expression system to allow for the investigation of the regulatory effect of one protein in the aggregation behaviour of the other. WT and mutant $\alpha \mathrm{S}$ and $\beta S$ were fluorescently tagged in their C-termini using a fast-folding version of GFP ("superfolder GFP" or sGFP) and mCherry, respectively (Figure 1A) and coexpressed in the cell-free translation reaction of the Leishmania Tarentolae Extracts (LTE) at different coexpression ratios for $3 \mathrm{~h}$ at $27^{\circ} \mathrm{C}$. By titrating the relative amount of DNA template used to prime the LTE, the final levels of expression of GFP- and Cherry-tagged proteins can be varied (Figure 1B). The relative expression levels between the two proteins can be rigorously controlled to obtain a fixed range of expression ratios of $\beta S: \alpha$ S varying between 0 for the $\alpha$ S C-sGFP controls and $\sim 1$ for the last coexpression tested, simulating naturally occurring similar expression levels between these two proteins (Figure 1B,C). To assess the aggregation propensity of $\alpha \mathrm{S} W T$ and 
mutants in the presence of different amounts of $\beta S$, we used single molecule spectroscopy. As shown by our group [37], single molecule techniques are well suited to the study of heterogeneous systems.

Briefly, the solution of GFP-tagged proteins at $\mathrm{nM}$ concentration is placed on a confocal microscope and fluorescence of GFP is recorded over time. As the fluorescent proteins diffuse in and out of the confocal volume due to Brownian motion, fluctuations around a "monomeric" average intensity are created. Larger fluorescent bursts correspond to larger aggregates, as is the case of the individually expressed $\alpha$ S C-GFP mutants (Figure 1D). The data can then be plotted as a distribution of brightness as in Figure 1E. In these plots, the contribution of the main species is represented as a Gaussian distribution and larger events are creating a tail in the distribution. The length of the tail is correlated with the maximum number of fluorophores in the objects, or to put it briefly, a longer tail indicates the presence of larger aggregates. As $\beta S$ is titrated into the coexpression system, we observe a reduction of the size of $\alpha \mathrm{S}$ aggregates (compare the black and grey curves on Figure 1E) indicating an inhibition of $\alpha S$ aggregation by $\beta S$. When a 1:1 ratio of $\beta S: \alpha S$ is present, $\alpha$ S becomes perfectly monomeric as shown by a typically monomeric fluorescent time trace (Figure 1D), as well as a purely Gaussian distribution of the brightness plot (Figure 1E, light grey curve).

\section{2. $\beta$-Synuclein Is a Selective Inhibitor of Different Aggregation Pathways of $\alpha$-Synuclein}

Having established a suitable experimental setup for assessing the aggregation of fluorescently tagged synucleins, we then set out on systematically investigating how $\beta S$ affects the behaviour of $\alpha \mathrm{S}$, by using the pathological aggregation-prone $\alpha \mathrm{S}$ mutants as a model for rapid access to different aggregated species.

As reviewed elsewhere [37] and explained in detail in the "Material and Methods" section, in single molecule spectroscopy, the 'brightness parameter- $\mathrm{B}^{\prime}$ ' is an ideal tool to monitor the aggregation of proteins, representing a measure of the heterogeneity of the sample in a concentration-independent manner. Moreover, when normalized to the brightness of the GFP monomer, B provides information on the size of the aggregated species. By acquiring $30 \mathrm{~s}$ fluorescent time traces in triplicate on four different independent measurements for each $\alpha \mathrm{S}$ (WT, A30P, E46K, H50Q, G51D and A53T), we were able to generate "aggregation-inhibition curves" across a range of expression ratios C-mCherry-tagged- $\beta S$ :C-sGFP-tagged- $\alpha$ S between 0 and approximately 1 (Figure 2A). The B parameter therefore complements the fluorescent time traces acquired for these ratios (Figure $2 \mathrm{~B}$ ), all of which paint a clear picture of $\beta S^{\prime}$ s chaperoning activity on different forms of $\alpha \mathrm{S}$.

As shown by our group before [22], the controls for each single-point mutant show a segregation into two different groups, with E46K, A53T and H50Q forming larger fibrils, whereas A30P and G51D tend to form smaller objects and WT is mainly monomeric. Remarkably, titration of $\beta S$ WT into the different coexpression systems results in distinct inhibitory effects. In fact, A30P and G51D "react" much more readily to even low titrations of $\beta S$, with very few aggregated forms being detected at $\beta S: \alpha S$ ratios above $\sim 0.2$. Conversely, the fibril-forming mutants E46K, H50Q and A53T are, up to a certain ratio $\beta S: \alpha S$, partly unaffected in their aggregation propensity. In fact, even 1:1 $\beta S: \alpha S$ ratios are not sufficient to rescue these mutants to the monomeric level. 
(A)

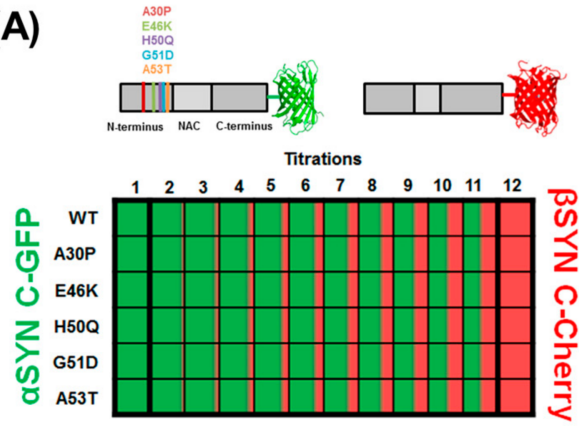

(B)

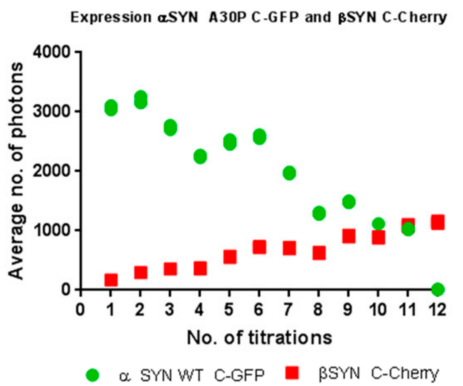

(C)

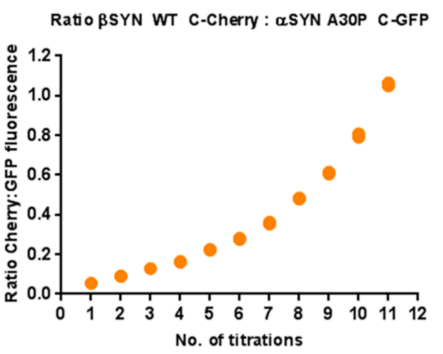

(D)

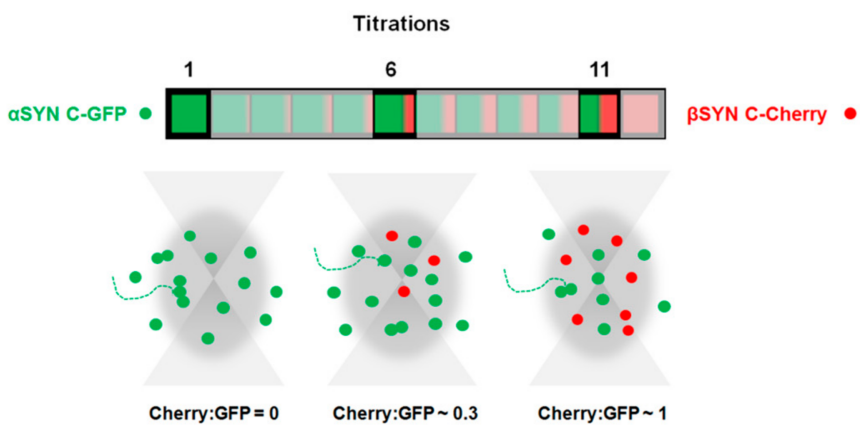

(E)

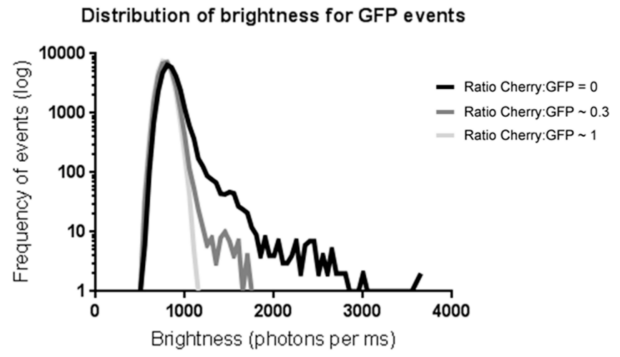

Figure 1. Controlled cell-free co-expression of $\alpha$ - and $\beta$-synucleins allows the investigation of their co-oligomerization dynamics. (A) Experimental layout for controlled co-expression of C-sGFP-tagged $\alpha$-Synucleins (WT and five PD-related pathological mutants A30P, E46K, H50Q, G51D and A53T) and C-mCherry-tagged $\beta$-synuclein WT. All proteins were expressed in LTE. (B,C) This experimental layout allows us to accurately control the expression levels of sGFP- and mCherry-tagged proteins and their ratio. (D) This translates into different relative amounts of proteins being detected as they diffuse in and out of confocal volume, creating different GFP-fluorescent time traces for each titration between $\alpha$ - and $\beta$-synuclein (see examples of titrations 1, 6 and 11). (E) Analysis of these traces as a function of brightness (photons per ms) shows the effect of $\beta$-synuclein on the oligomerization propensity of $\alpha$-synucleins. 
(A)
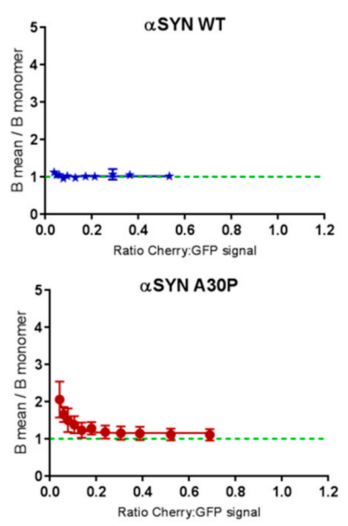

$\alpha$ SYN E46K

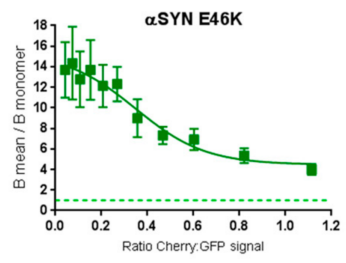

$\alpha S Y N$ H50Q

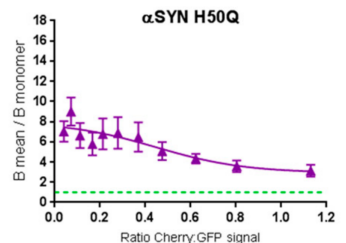

$\alpha$ SYN G51D
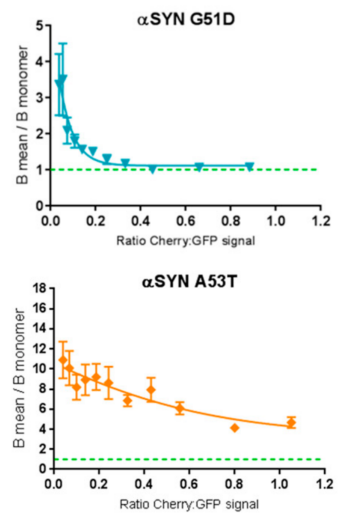

(B)

(i)

(ii)

(iii)
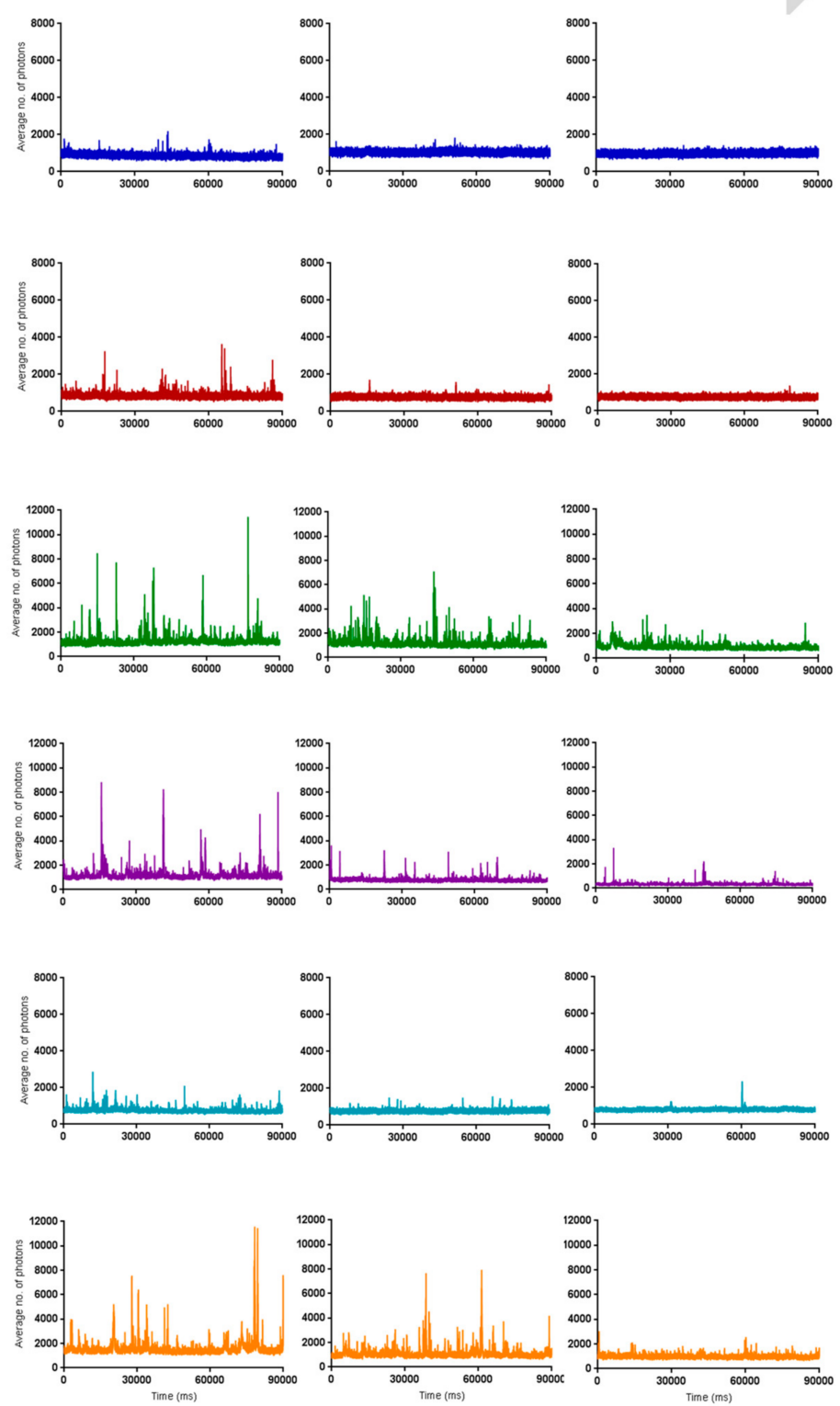

Figure 2. Brightness analysis of $\alpha$-synuclein mutants reveals selective inhibition of their pathological aggregation by $\beta$-synuclein. (A) The brightness parameter was calculated for the GFP tagged proteins and plotted against the the mCherry $\beta$-synuclein: sGFP $\alpha$-synuclein ratio $(0<$ ratio Cherry:GFP $<1.2)$. For each ratio, three $30 \mathrm{~s}$ replicates were acquired and averaged for a total of four co-expression experiments for each protein. The brightness of the GFP monomer is indicated by a green dashed line. Error bars represent the SE of those 4 independent measurements. (B) GFP fluorescence time traces show the inhibition of $\alpha$-synuclein aggregation from (i) the initial control C-terminal sGFP-tagged $\alpha$-syn, to (ii) 1:3 of $\beta$ syn-mCherry: $\alpha$ syn-sGFP and, finally (iii) 1:1 Cherry:GFP. Arrow indicates this increase in $\beta: \alpha$ ratio.

\subsection{Preferential Binding to Smaller Oligomers Determines a More Efficient Inhibition of Aggregation}

To explore the mechanism underlying the selective inhibition of some of the $\alpha \mathrm{S}$ mutants' aggregation by $\beta S$, we examined the hypothesis that the observed inhibition is the result of different affinities to different aggregated species. To this end, we performed two-color coincidence 
measurements. In this experiment, two lasers, exciting in the GFP and Cherry wavelengths, are focused in the same confocal volume and fluorescence of the two fluorophores is detected separately. The detection of co-diffusion between GFP and Cherry in the fluorescent bursts indicates the presence of the two species in the same object (Figure 3A). The stoichiometry of the interaction can be quantified as the coincidence ratio $\left(C=\frac{I_{\text {Cherry }}}{I_{\text {GFP }}+I_{\text {Cherry }}}\right)$ (Figure 3B).

(A)
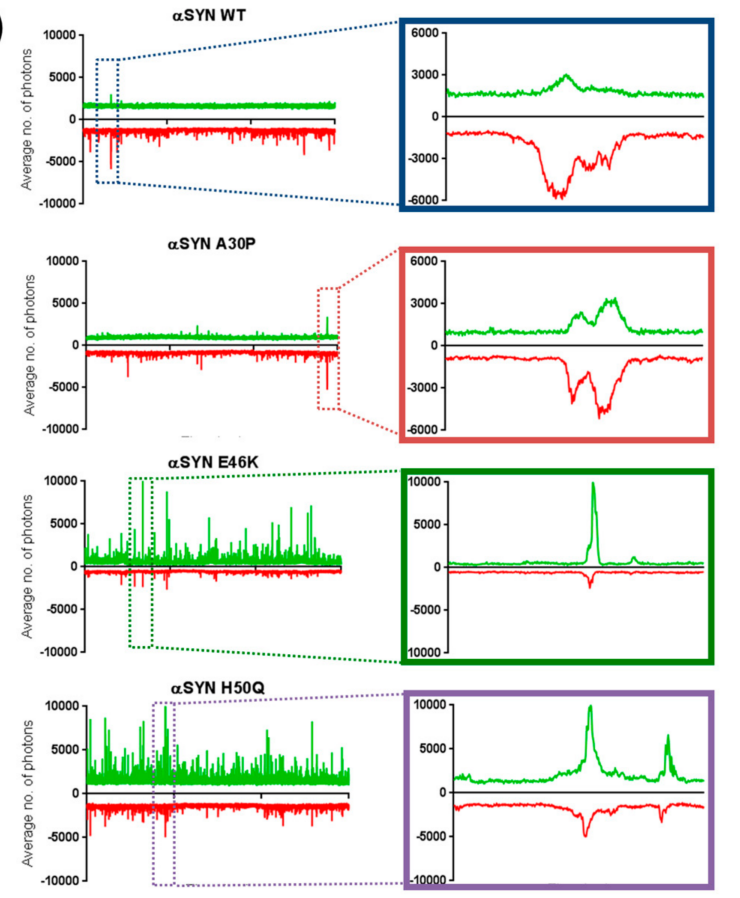

$\alpha$ SYN G51D

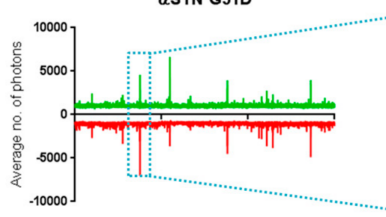

$\alpha$ SYN A53T

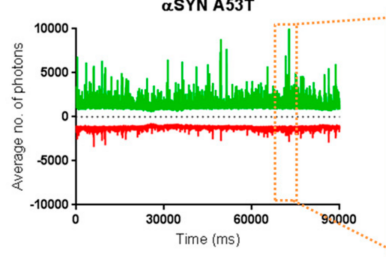

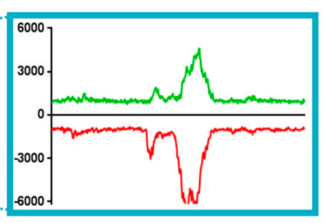

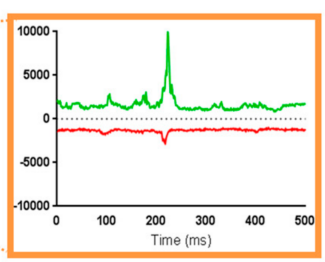

(B)
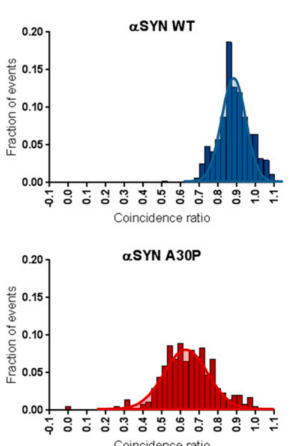

$\alpha$ SYN E46K

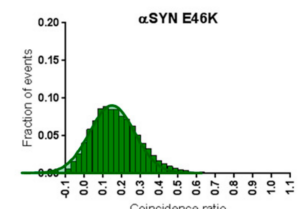

$\alpha$ SYN H50Q

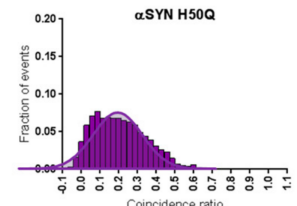

$\alpha$ SYN G51D

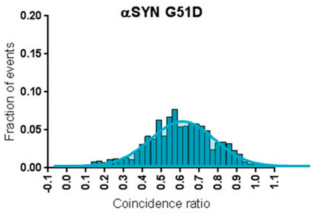

$\alpha$ SYN A53T

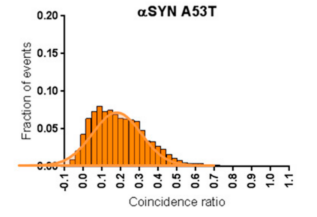

Figure 3. Two-color coincidence unveils the selective mechanism by which $\beta$-synuclein inhibits the aggregation of the different $\alpha$-synuclein mutants. (A) For systematic coincidence measurements, only $1: 1(\beta: \alpha), 90 \mathrm{~s}$ traces were selected and three measurements were performed for each protein using a scanning-well microscope approach. Examples of traces and the detailed fluorescent bursts used for coincidence plots show the different trend across the different pathological mutants. (B) Coincidence ratios between the traces of mCherry $\beta$-syn WT and sGFP $\alpha$-syns were plotted for a total number of at least 400 events. For each trace, the threshold for an "event" was defined as any burst $\geq$ average expression + standard deviation of pure GFP monomer.

To understand how this co-diffusion unfolds, all two-color coincidence measurements were first performed at 1:1 ratio of mCherry and sGFP proteins. However, as shown in Figure 2B, in our experimental setup, to work at these $\beta S: \alpha S$ expression ratios means detecting mostly monomeric form of the C-GFP $\alpha \mathrm{S}$ due to the inhibitory effect performed by $\beta S$. To solve this problem, the confocal 
microscope was adapted to automatically scan the wells of the measuring plate during data acquisition and, in this way, retrieve the rarer aggregates that might be missed when relying on Brownian motion. As a result, more aggregates are detected over the course of our $30 \mathrm{~s}$ traces, allowing us to collect enough fluorescent events for two-color coincidence measurements.

In respect to the six different forms of $\alpha$ S tested against $\beta S W T$, fluorescence time traces show more co-diffusion of mCherry-tagged $\beta S$ with the sGFP-tagged oligomers of $\alpha \mathrm{S}$ A30P and G51D as opposed to with the larger events originated by E46K, H50Q and A53T (Figure 3A). To quantify this effect, all bursts of fluorescence above threshold, corresponding to oligomers, were analysed for coincidence and histograms of distribution of the coincidence ratios (C) were plotted (Figure 3B). These histograms can be used to a measure of the stoichiometry of the interactions. Briefly, a distribution centred on $\mathrm{C}=0$ indicates the presence of oligomers containing only GFP-tagged proteins while a distribution around $\mathrm{C}=1$ reflects the presence of Cherry-tagged oligomers/aggregates. The presence of a population at different $C$ values indicates that co-aggregation is possible and the average $C$ value calculates the average stoichiometry of the assembly. The population of A30P and G51D aggregates shows a considerable shift in this average when compared to the other 3 mutants from $\sim 0.6$ to $\sim 0.15$, respectively. This indicates a clear propensity of $\beta S$ to co-aggregate with A30P and G51D while being mainly excluded from the E46K/H50Q/A53T aggregates. Indeed, on average, for each A30P/G51D aggregate that diffuses through the confocal volume, a similar number of $\beta S$ molecules is detected, whereas, for $\mathrm{E} 46 \mathrm{~K} / \mathrm{H} 50 \mathrm{Q} / \mathrm{A} 53 \mathrm{~T}$, this number is four times smaller.

We then performed the same two-color coincidence analysis to all $90 \mathrm{~s}$ traces across the different $\beta S: \alpha S$ titrations and plotted the averages of the distribution of coincident events against the ratio $\beta S: \alpha S$ (Figure 4). The threshold for an "event" was defined as the average expression plus the standard deviation of the GFP monomer (Figure S1). Results validated the high propensity for $\beta S$ to incorporate into aggregates from $\mathrm{A} 30 \mathrm{P}$ and G51D, even for low expression levels of $\beta S$, whereas other $\alpha \mathrm{S}$ aggregated forms are relatively unaffected.

(A)
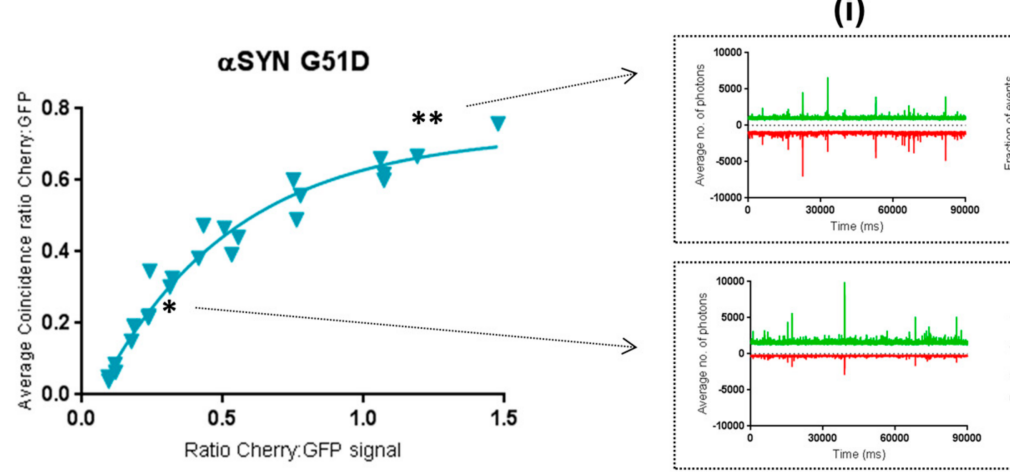

(ii)
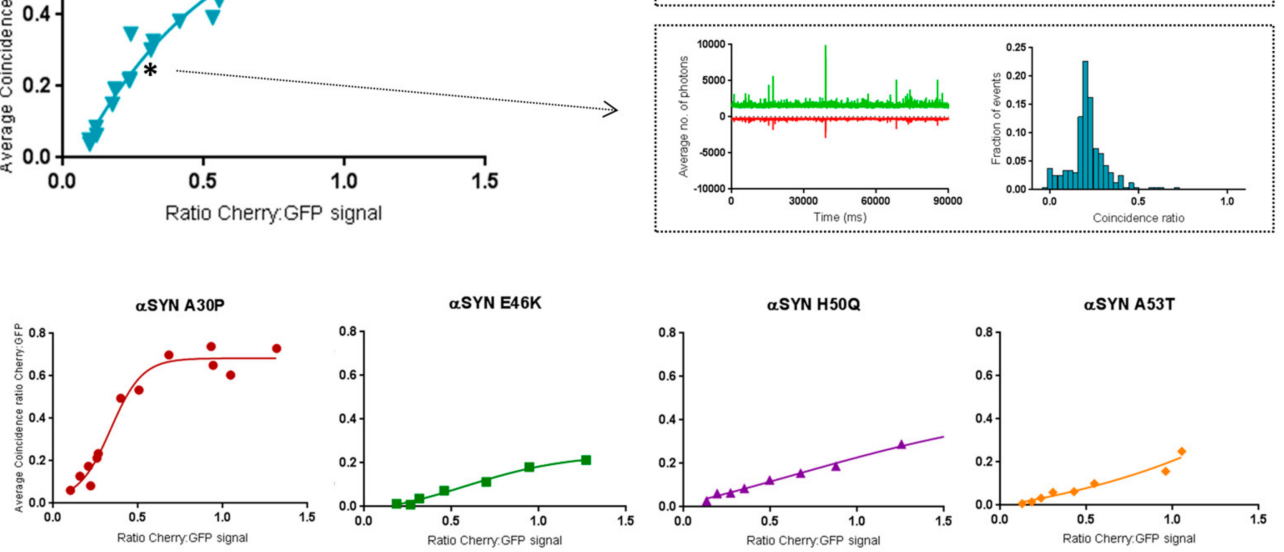

Figure 4. Two-color coincidence applied to the $\beta: \alpha$ titration range validates affinity differences between aggregated forms of synucleins. (A) Two-color coincidence analysis was performed for all $90 \mathrm{~s}$ traces (i) corresponding to the different concentration ratios tested. The distributions of coincident events (ii) were averaged and averages of coincidence ratios were plotted across the $\beta: \alpha$ ratios as exemplified by $\alpha \mathrm{S}$ G51D ("* $\rightarrow$ " ratio $=0.25$; and "** $\rightarrow$ " ratio $=1.2)$. (B) The same plots were acquired for all mutant forms of $\alpha \mathrm{S}$ revealing two types of behaviours. 


\section{4. $\beta$-Synuclein Does Not Bind to the Aggregates of $\alpha$-Synuclein Mutants}

We next asked whether this co-diffusion reflects co-aggregation with incorporation of $\beta S$ in $\alpha S$ aggregates during elongation or binding of $\beta S$ to already-formed $\alpha \mathrm{S}$ aggregates.

To test whether this was due to incorporation of $\beta S$ in $\alpha$ S aggregates, we then individually expressed the same proteins for $3 \mathrm{~h}$ before mixing them to obtain similar final expression ratios. Figure 5 depicts the results obtained for three of the five mutants, representing the two groups. Results showed that aggregation propensity (indicated by B) of A30P, H50Q and G51D is relatively unchanged when $\beta S$ and the mutant $\alpha$-synucleins are expressed separately (Figure 5A). Furthermore, fluorescent time traces show little coincidence in all cases (Figure 5B), translating into distributions of coincidence ratio shifted towards 0 (i.e., GFP-only objects) (Figure 5C). In particular, we noted a shift in the distribution of coincidence ratios for A30P and G51D as indicated in Figure 5C (top and bottom panels). Established A30P/G51D $\alpha \mathrm{S}$ oligomeric assemblies are undisturbed by the addition of $\beta S$ attesting the inefficacy of $\beta S$ to modulate $\alpha S^{\prime}$ s aggregation beyond the earlier steps of oligomerization. These aggregation-prone mutants, here represented by $\mathrm{H} 50 \mathrm{Q}$, do not present this shift in their coincidence plots, indicating that $\beta S$ plays a limited role in regulating their aggregation cascade. With this approach, we were able to uncover a selectivity of $\beta S$ to incorporate and modulate further assembly of specific oligomeric forms of $\alpha \mathrm{S}$.

(A)

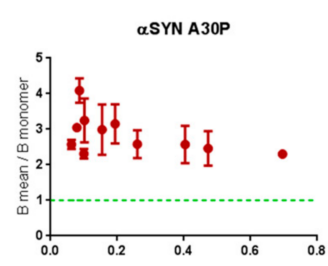

$\alpha S Y N$ H50Q

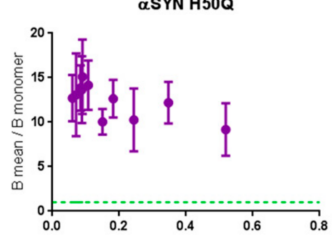

$\alpha$ SYN G51D

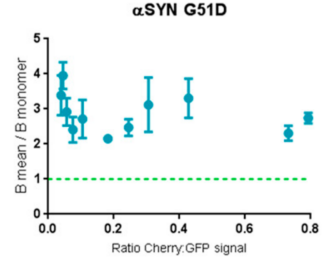

(B)

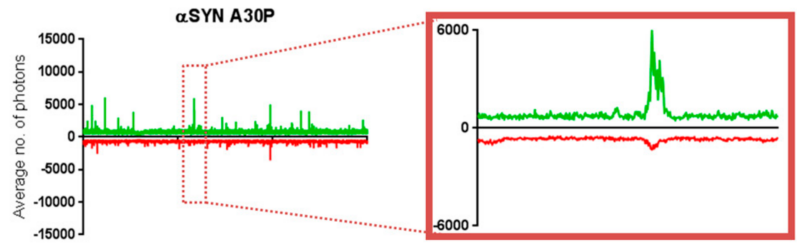

$\alpha S Y N ~ H 50 Q$

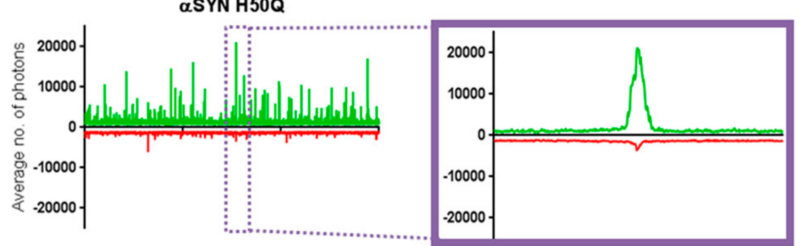

$\alpha$ SYN G51D
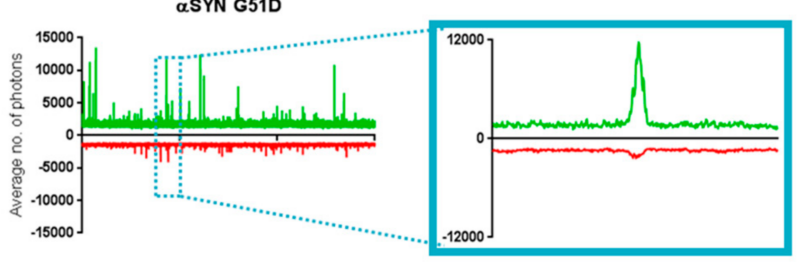

(C)
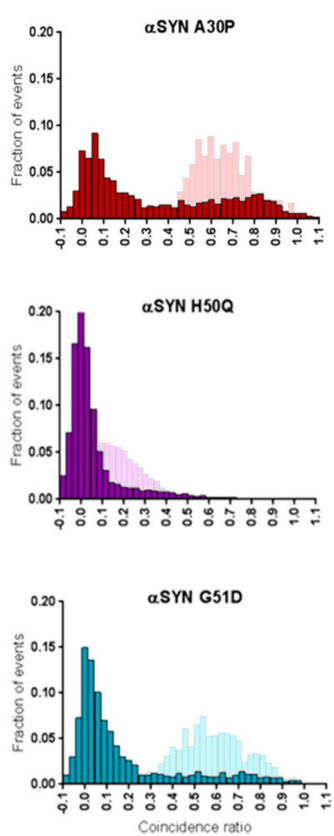

Figure 5. Individual expression of proteins prior to mixing irrevocably reveals a model of selective incorporation of $\beta S$ into specific $\alpha S^{\prime}$ s aggregated species. (A) Brightness analysis averaged plots were acquired for three independent measurements of triplicated $30 \mathrm{~s}$ fluorescent time traces using a stationary microscope plate setup. Error bars represent the SE of those 3 independent measurements Here mutants A30P, H50Q and G51D are shown for final $\beta S W T(C h e r r y): \alpha S(G F P)$ ratios $<0.8$. Brightness values are normalized against the brightness of the GFP monomer (green dashed line). (B) 90-s fluorescent time traces were acquired by scanning confocal microscopy and $(\mathbf{C})$ two-color coincidence was applied to a population of $>400$ fluorescent bursts. Coincidence histograms (dark colour) were compared with the ones obtained from coexpression of the same proteins (lighter). 


\section{Discussion}

Ever since the discovery of different members in the synuclein family, important research has aimed at understanding how these highly homologous proteins might relate in function. Much evidence has thereafter surfaced supporting the idea that $\beta S$ plays a role in the inhibition of $\alpha S$ aggregation, but the mechanistic basis and exact stage in $\alpha S^{\prime}$ s aggregation when this occurs is still unclear. In earlier work, we have demonstrated that single molecule fluorescence methods are a useful tool to quantify protein oligomerization [37] and enable us to dissect the events leading to the formation of fibrils in $\alpha \mathrm{S}$ pathology [22]. In the present study, we have investigated the effect of co-expressing WT and mutant $\beta$ - and $\alpha$-synucleins in our cell-free system. By applying single molecule brightness analysis and two-color coincidence, we were able to show that $\beta S$ inhibits the aggregation of $\alpha S$ with different degrees of efficiency depending on the mutant $\alpha S$ tested. In our cell-free system, where we study proteins within a few hours after expression at low concentrations, we observe smaller oligomers formed by A30P and G51D and larger aggregates and protofibrils formed by E46, H50Q and A53T and see segregation into different classes [22]. Therefore, we formulated the hypothesis that the different point mutants represent distinct $\alpha \mathrm{S}$ aggregated species and specific oligomerisation/fibrillation steps. Consequently, by investigating how $\beta S$ modulates the aggregation of $\alpha S$ mutants, we are interrogating its impact on the formation of early oligomers in the aggregation pathway.

Taken together, our data enabled us to construct a proposed model for the chaperoning effect of $\beta S$ on $\alpha \mathrm{S}$, depicted in Figure 6. Our results point to a more efficient inhibition of aggregation for oligomer-forming A30P and G51D as compared to E46K, H50Q and A53T. This 'resistance' to $\beta S^{\prime}$ 's inhibitory effect shown by the latter class of mutants has not been described before and effectively assigns a selective inhibitory effect of $\beta S$ towards the earlier steps of oligomerisation in the pathway.

$\beta S$ and $\alpha S$ seem to be interchangeable in the early oligomers, as shown by the two-color coincidence data of Figures 3 and 4 . As the ratio $\beta: \alpha$ in the system increases, $\beta S$ replaces $\alpha S$ in the small oligomers in a concentration-dependent manner. The gradual shielding of $\alpha \mathrm{S}-\alpha \mathrm{S}$ interactions inhibits their self-assembly and ultimately the oligomers of $\alpha S$ can no longer form. At the same time, as $\beta S$ does not aggregate readily, the overall number of aggregated species decrease with $\beta S$ concentration.

The inhibition effect occurs very quickly for A30P and G51D, and cannot be attributed to reductions in protein expression levels. In a previous work [22], we have shown that cell-free expressed synucleins mutants displayed aggregation at low concentrations. All mutants showed very stable brightness parameter over an order of magnitude or more, down to $100 \mathrm{nM}$ expression levels. In this work, the co-expression with $\beta S$ only reduces protein expression by a factor 2 to 3 .

One important aspect of our experiments is that $\alpha S$ and $\beta S$ can co-oligomerize only when co-expressed. As shown in Figure 4, $\beta S$ can no longer incorporate into the pre-formed $\alpha$ S oligomers. This indicates that co-translational association and fast formation of oligomers is crucial and that oligomers are stable in composition: once trapped in an oligomer, the proteins do not exchange with monomers present in solution.

Our study utilises the relatively large sGFP and mCherry fluorescent tags and we performed extensive checks to make sure that these tags do not interfere with our measurements. This is especially important for synucleins, as the tags are larger than the protein itself. sGFP and mCherry could either block the aggregation of the proteins due to steric effects, or create false-positives interactions if sGFP and mCherry could interact in our experimental conditions. In our previous work [22], we used a much smaller $6 \times$ histidine tag and checked that the GFP label did not affect the aggregation propensity of the proteins. We used a Tris-NTA coupled dye to label the oligomers after their expression in our cell-free system and could not find differences in the aggregation properties measured [22]. In the same study, we demonstrated that the sGFP and mCherry tags did not interfere with binding of $\alpha$-synucleins, as we measured protein-protein interactions using AlphaScreen methods. We also performed coincidence measurements, both at the dimer level and at the oligomer level. This demonstrates that the mCherry tag is not responsible for inhibition of oligomerisation. The complete absence of interaction between WT and some synuclein mutants also proves that the sGFP and mCherry tags 
do not bind to each other in our experimental conditions, creating false positives. This is expected as these fluorescent tags are now widely used for in cell studies and chaperoning or aggregation effects would have been described.

In our system, we observe that the mutants E46K, H50Q and A53T seem to "skip" the oligomerisation step, or at least transition quickly to pre-fibrillar species. Our data show that $\beta S$ cannot incorporate efficiently into the aggregates of these mutants, as indicated by the very low coincidence ratios (Figures 3 and 4). We also show that $\beta S$ cannot efficiently block the aggregation of the E46K, H50Q and A53T mutants as we observe a smaller decrease of aggregation propensity in Figure 2. In that case, $\alpha S$ can fibrillate efficiently on its own, $\beta S$ is less likely to be recruited, and, logically, $\beta S$ can no longer interfere with fibrillation and cannot block the elongation of fibrils. In other words, for these $\alpha \mathrm{S}$ mutants, aggregation is inhibited as well, but it becomes only significant when the concentration balance between available $\alpha$ S and $\beta S$ molecules leans to the latter. These findings are consistent with the hypothesis, postulated by other authors, that $\beta S$ acts a natural retardant of $\alpha \mathrm{S}$ aggregation by competing with $\alpha \mathrm{S}$ molecules for fibril amplification [33]. To put it simply, our data suggest that $\beta S$ recognizes the monomeric or oligomeric form of $\alpha S$ but not the fibrillar structure. This is reminiscent of our previous observations that WT $\alpha$ S cannot associate efficiently with the mutants E46K, H50Q and A53T [22]. In many ways, $\beta S$ behaves as WT $\alpha$ S at the monomeric and oligomeric level.

Other authors have assessed the binding affinity and characteristics of $\beta S$ WT to $\alpha S$ WT, showing that both directly interact at the monomer level to form transient heterodimers with high specificity [25]. NMR experiments have demonstrated that interactions between the $\mathrm{N}$-terminus of $\alpha \mathrm{S}$ and the C-terminus of $\beta S$ (forming non-propagating heterodimers) are five times stronger and more extensive than those of the $\mathrm{C}$-terminus of $\alpha \mathrm{S}$ with its own $\mathrm{N}$-terminus (propagating homodimers that proceed to fibril formation). This could provide us with an explanation as to why the aggregation of A30P and G51D is more efficiently inhibited. Seeing that these two mutants were shown to recruit WT $\alpha \mathrm{S}$ [22], if we add $\beta S$ into the system, competition for binding could determine delay in aggregation. Here, our study of the $\alpha \mathrm{S}$ mutants completes the picture of $\alpha \mathrm{S}-\beta S$ interactions.

However, further fundamental biological questions surface from the model we propose here. One would reasonably interrogate whether this putative delay in aggregation exerted by $\beta S$ also presents an effect in terms of overall toxicity of the 'equilibrium' of aggregated species present in the system at any given moment. Moreover, if that is the case, would the regulatory action exerted by $\beta S$ favour or abrogate toxicity? In fact, oligomers of G51D $\alpha$ S have been shown to be more toxic to cells and the disease caused by G51D mutation to progress faster [38,39]. Recent years have strengthened the views that implicate interactions with proteasome subunits in $\alpha \mathrm{S}$ pathology. It has been postulated that $\beta S^{\prime}$ s role as a negative regulator of $\alpha S$ is related to a competitive interaction with $\alpha S$ aggregates, which would make them less available for recognition by the $26 \mathrm{~S}$ subunit of the proteasome system [40]. This could point to a close functional synergy between $\beta S$ and the degradation pathways in proteostasis, where $\beta S$ could act as a filter for other degradation pathways. Strikingly, despite the multiple lines of evidence that prove presynaptic colocalization, regulatory effects and interactions between $\alpha \mathrm{S}$ and $\beta S$, these proteins do not coincide in Lewy body inclusions [41]. Our in vitro data on E46K, H50Q and A53T are consistent with this physiological observation as $\beta$ S does not associate with their aggregates (Figure 4). If the presence of protofibrils of $\alpha S$ remains to be demonstrated in the brain, our study highlights a differential recognition of $\alpha$ S species by $\beta S$ and suggests different interactomes for the different aggregation "steps" of $\alpha \mathrm{S}$. 


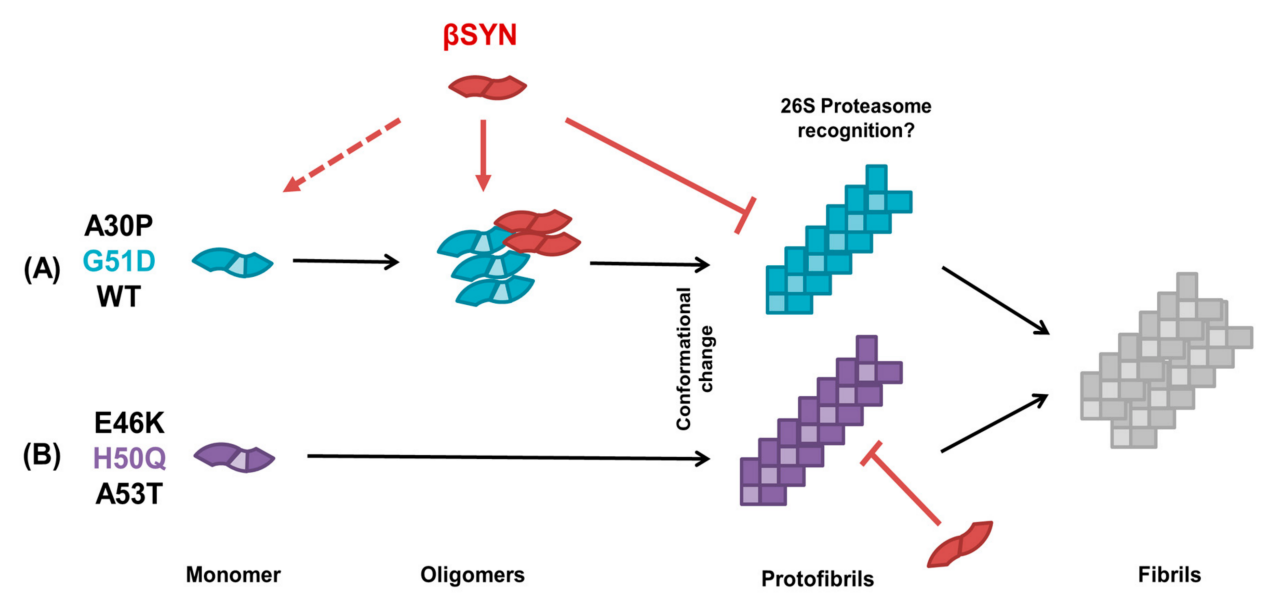

Figure 6. Proposed model of modulation of $\alpha$-synuclein aggregation by its putative "natural negative regulator" $\beta$-synuclein. The model shows two distinct pathways of aggregation for the different $\alpha \mathrm{S}$ mutants, in (A) and (B). $\alpha \mathrm{S}$ monomers are depicted as blue and purple coloured blocks with a light-coloured core representing the NAC domain, which is absent in $\beta S$, represented by red blocks. Arrows represent the proposed model's sequence; dashed lines represent discussion points in this model for future studies; capped ends depict no interaction. Monomeric and oligomeric forms are represented unfolded, and a conformational change is required for $\alpha$-synuclein to display pre-fibrillar and fibrillar species.

\section{Materials and Methods}

\subsection{Preparation of LTE}

Leishmania tarentolae cell-free lysate was produced as described by Johnston and Alexandrov [42-44]. Briefly, Leishmania tarentolae Parrot strain was obtained as a LEXSY host P10 from Jena Bioscience GmbH, Jena, Germany and cultured in TBGG medium containing $0.2 \% v / v$ Penicillin/Streptomycin (Life Technologies, Carlsbad, CA, USA) and 0.05\% $w / v$ Hemin (MP Biomedicals, Seven Hills, NSW, Australia). Cells were harvested by centrifugation at $2500 \times g$, washed twice by resuspension in $45 \mathrm{mM}$ HEPES buffer, $\mathrm{pH}$ 7.6, containing $250 \mathrm{mM}$ Sucrose, $100 \mathrm{mM}$ Potassium Acetate and $3 \mathrm{mM}$ Magnesium Acetate and resuspended to $0.25 \mathrm{~g}$ cells $/ \mathrm{g}$ suspension. Cells were placed in a cell disruption vessel (Parr Instruments, Moline, IL, USA) and incubated under $7000 \mathrm{KPa}$ nitrogen for $45 \mathrm{~min}$, and then lysed by rapid release of pressure. The lysate was clarified by sequential centrifugation at $10,000 \times g$ and $30,000 \times g$ and anti-splice leader DNA leader oligonucleotide was added to $10 \mu \mathrm{M}$. The lysate was then desalted into $45 \mathrm{mM}$ HEPES, pH 7.6, containing, $100 \mathrm{mM}$ Potassium Acetate and $3 \mathrm{mM}$ Magnesium Acetate, supplemented with a coupled translation/transcription feeding solution and snap-frozen until required.

\subsection{Gateway Cloning System for Cell-Free Protein Expression}

$\alpha$ - and $\beta$-synucleins DNA were synthesized by IDT (Coralville, IA, USA) as G-blocks and were cloned into the following cell free expression Gateway destination vectors, respectively: C-terminal sGFP tagged (pCellFree_G04) and C-terminal mCherry-cMyc tagged (pCellFree_G08) [45]. Transfer between destination vectors being carried using Gateway PCR cloning protocol, as described in [46].

\subsection{Cell-Free Coexpression and Fluorescence Spectroscopy of $\alpha$-and $\beta$-Synucleins}

Plasmids encoding C-mCherry- and C-sGFP-tagged proteins were expressed in LTE at a concentration DNA template to lysate of $40 \mathrm{nM}$ and $20 \mathrm{nM}$, respectively, and immediately mixed at different ratios to a final volume of $10 \mu \mathrm{L}$ across a range of twelve titrations (including two controls, one for individual expression of C-sGFP $\alpha S$ and one for C-mCherry $\beta S$ ). This experimental setup 
was equally applied to all mutant $\alpha$-synucleins, aiming at obtaining fixed ratios of expression levels of $\beta S$ to $\alpha$ S between 0 and 1 . Proteins were allowed to coexpress for $3 \mathrm{~h}$ at $27^{\circ} \mathrm{C}$ and then diluted in buffer A ( $25 \mathrm{mM}$ HEPES, $50 \mathrm{mM} \mathrm{NaCl})$. A volume of $20 \mu \mathrm{L}$ of each sample was placed into a custom-made 192-wells silicone plate with a $70 \times 80 \mathrm{~mm}$ glass coverslip (ProSciTech, Kirwan, QLD, Australia). Plates were analyzed at room temperature on a Zeiss Axio Observer microscope (Zeiss, Oberkochen, Germany) with a custom-built data acquisition setup. In order to gain a clear picture of the oligomerisation and aggregation of the proteins, we acquired time-traces at concentrations between 1 and 100 nanomolar (nM).

\subsection{Brightness Analysis}

For intensity measurements, the C-terminal sGFP-labelled proteins were expressed. A $488 \mathrm{~nm}$ laser beam was focused in the sample volume using a $40 \times / 1.2 \mathrm{NA}$ water immersion objective (Zeiss, Oberkochen, Germany). The fluorescence of sGFP was measured through a 525/20 nm band pass filter, and the number of photons collected in $1 \mathrm{~ms}$ time bins $(\mathrm{I}(\mathrm{t}))$ was recorded. The proteins were diluted 10 times in buffer A and fluorescent traces were acquired in triplicated measurements of $30 \mathrm{~s}$.

The fluorescent time-trace I $(\mathrm{t})$ obtained shows the presence of intense bursts of fluorescence, with values well over the typical fluctuations of $I(t)$. The presence of these bursts increases the standard deviation of the distribution. To compare the aggregation at different concentrations, we used the Brightness parameter, which is the standard deviation normalized by the average signal, as described previously [37]:

$$
B=\frac{S D^{2}}{\text { average }}
$$

The final brightness parameters acquired for each $\alpha \mathrm{S}$ represent averages of four independent measurements. Data was normalized against the brightness determined for sGFP across our working range of concentrations, and curve fitting was performed using GraphPad Prism version 7.00 (La Jolla, CA, USA) for Windows.

\subsection{Two-Color Coincidence Measurements}

For coincidence experiments, sGFP- and mCherry-labelled proteins were either co-expressed or expressed separately and mixed immediately prior to data acquisition. Fluorescent time traces were acquired for $30 \mathrm{~s}$ with a scanning confocal microscope, which scans each well of the microscope plate in different directions for $30 \mathrm{~s}$ in triplicate, allowing us to retrieve data on the rarer aggregates that diffuse more inefficiently. For stoichiometric purposes, this procedure was optimized so that final expression levels were 1:1. In this context, average fluorescence was similar in the two channels, and we were able to use the same definition of a "fluorescent event" for both GFP and Cherry. We defined a fluorescent burst as any fluorescence intensity above the sum of the average intensity with the standard deviation of sGFP, acquired for our concentration range. For each event, the intensities of the GFP and Cherry bursts were corrected for background and leakage (6\% leakage of the GFP intensity into the Cherry channel).

The coincidence $\mathrm{C}$ was then measured as the corrected Cherry signal $\left(\mathrm{I}_{\mathrm{C}}\right)$, divided by the total intensity of the burst $\left(\mathrm{C}=\mathrm{I}_{\mathrm{C}} /\left[\mathrm{I}_{\mathrm{G}}+\mathrm{I}_{\mathrm{C}}\right]\right)$. In the absence of Cherry fluorescence, $\mathrm{C}$ is close to zero, while, in the absence of GFP, $C$ tends towards 1 . Events with $0.25<\mathrm{C}<0.75$ are considered coincident events. The number of events for each ratio $C$ was counted and normalized to the total number of events to give a probability $\mathrm{P}(\mathrm{C})$. Histograms of single-molecule coincidence $(\mathrm{P}(\mathrm{C})$ as a function of $\mathrm{C})$ were obtained by measuring >1000 events per interaction, and fitted by Gaussian peaks for GFP-only, coincidence and Cherry-only contributions. The bound fraction was calculated as the proportion of coincidence $(0.25<\mathrm{C}<0.75)$ to total events. 


\section{Conclusions}

To conclude, these findings attest to the potential for using single molecule techniques to deconstruct an $\alpha \mathrm{S}$ aggregation cascade, especially when utilized in conjunction with an experimental design that includes models for different aggregated species along that cascade (pathological mutants of $\alpha \mathrm{S}$ ) and inhibitors of that process ( $\beta$-Synuclein).

Supplementary Materials: Supplementary materials can be found at www.mdpi.com/1422-0067/19/2/334/s1. Acknowledgments: This work was supported by grants from the National Health and Medical Research Council of Australia (project grants APP1108859 to Yann Gambin and Emma Sierecki, APP1120374 to Emma Sierecki). Yann Gambin was supported by an Australian Research Council Future Fellowship (FT110100478) during this project.

Author Contributions: Andre Leitao carried out the coexpression, oligomerization and two-color coincidence experiments. Andre Leitao and Yann Gambin performed brightness data acquisition as well as two-color coincidence data analysis. Andre Leitao, Yann Gambin and Emma Sierecki designed the study and drafted the manuscript. Dominic J.B. Hunter carried out LTE production. Akshay Bhumkar performed lysate optimization for $\alpha$ - and $\beta$ S expression and assisted with gateway cloning.

Conflicts of Interest: The authors declare no conflict of interest.

\section{Abbreviations}

$\begin{array}{ll}\alpha \mathrm{S} & \alpha \text {-Synuclein } \\ \alpha \mathrm{SYN} & \alpha \text {-Synuclein } \\ \beta S & \beta \text {-Synuclein } \\ \beta S Y N & \beta \text {-Synuclein } \\ \text { PD } & \text { Parkinson's Disease } \\ \text { CNS } & \text { Central Nervous System } \\ \text { DLB } & \text { Dementia with Lewy Bodies } \\ \text { IDP } & \text { Intrinsically Disordered Protein } \\ \text { BP } & \text { Brightness parameter } \\ \text { GFP } & \text { Green Fluorescent Protein } \\ \text { sGFP } & \text { "superfolder" Green Fluorescent Protein } \\ \text { mCherry } & \text { Monomeric Cherry } \\ \text { LTE } & \text { Leishmania Tarentolae Extract } \\ \text { SD } & \text { Standard Deviation } \\ \text { SE } & \text { Standard Error } \\ \text { WT } & \text { Wild-type } \\ \text { LTE } & \text { Leishmania tarentolae extract } \\ \text { HEPES } & \text { 4-(2-hydroxyethyl)-1-piperazineethanesulfonic acid }\end{array}$

\section{References}

1. Spillantini, M.G.; Schmidt, M.L.; Lee, V.M.-Y.; Trojanowski, J.Q.; Jakes, R.; Goedert, M. $\alpha$-Synuclein in Lewy bodies. Nature 1997, 388, 839-840. [CrossRef] [PubMed]

2. Goedert, M.; Spillantini, M.G.; Del Tredici, K.; Braak, H. 100 years of Lewy pathology. Nat. Rev. Neurol. 2013, 9, 13-24. [CrossRef] [PubMed]

3. Burré, J. The synaptic function of $\alpha$-synuclein. J. Park. Dis. 2015, 5, 699-713. [CrossRef] [PubMed]

4. Burré, J.; Sharma, M.; Tsetsenis, T.; Buchman, V.; Etherton, M.R.; Südhof, T.C. $\alpha$-Synuclein promotes SNARE-complex assembly in vivo and in vitro. Science 2010, 329, 1663-1667. [CrossRef] [PubMed]

5. Alderson, T.R.; Markley, J.L. Biophysical characterization of $\alpha$-synuclein and its controversial structure. Intrinsically Disord. Proteins 2013, 1, 18-39. [CrossRef] [PubMed]

6. Winner, B.; Jappelli, R.; Maji, S.K.; Desplats, P.A.; Boyer, L.; Aigner, S.; Hetzer, C.; Loher, T.; Vilar, M.; Campioni, S. In vivo demonstration that $\alpha$-synuclein oligomers are toxic. Proc. Natl. Acad. Sci. USA 2011, 108, 4194-4199. [CrossRef] [PubMed] 
7. Cremades, N.; Cohen, S.I.; Deas, E.; Abramov, A.Y.; Chen, A.Y.; Orte, A.; Sandal, M.; Clarke, R.W.; Dunne, P.; Aprile, F.A. Direct observation of the interconversion of normal and toxic forms of $\alpha$-synuclein. Cell 2012, 149, 1048-1059. [CrossRef] [PubMed]

8. Hoyer, W.; Cherny, D.; Subramaniam, V.; Jovin, T.M. Impact of the acidic C-terminal region comprising amino acids 109-140 on $\alpha$-synuclein aggregation in vitro. Biochemistry 2004, 43, 16233-16242. [CrossRef] [PubMed]

9. Gallea, J.I.; Celej, M.S. Structural insights into amyloid oligomers of the Parkinson Disease-related protein $\alpha$-Synuclein. J. Biol. Chem. 2014, 289, 26733-26742. [CrossRef] [PubMed]

10. Danzer, K.M.; Krebs, S.K.; Wolff, M.; Birk, G.; Hengerer, B. Seeding induced by $\alpha$-synuclein oligomers provides evidence for spreading of $\alpha$-synuclein pathology. J. Neurochem. 2009, 111, 192-203. [CrossRef] [PubMed]

11. Lee, H.-J.; Suk, J.-E.; Patrick, C.; Bae, E.-J.; Cho, J.-H.; Rho, S.; Hwang, D.; Masliah, E.; Lee, S.-J. Direct transfer of $\alpha$-synuclein from neuron to astroglia causes inflammatory responses in synucleinopathies. J. Biol. Chem. 2010, 285, 9262-9272. [CrossRef] [PubMed]

12. Iljina, M.; Garcia, G.A.; Horrocks, M.H.; Tosatto, L.; Choi, M.L.; Ganzinger, K.A.; Abramov, A.Y.; Gandhi, S.; Wood, N.W.; Cremades, N. Kinetic model of the aggregation of $\alpha$-synuclein provides insights into prion-like spreading. Proc. Natl. Acad. Sci. USA 2016, 113, E1206-E1215. [CrossRef] [PubMed]

13. Narkiewicz, J.; Giachin, G.; Legname, G. In vitro aggregation assays for the characterization of $\alpha$-synuclein prion-like properties. Prion 2014, 8, 19-32. [CrossRef] [PubMed]

14. Li, J.; Uversky, V.N.; Fink, A.L. Effect of familial Parkinson's disease point mutations A30P and A53T on the structural properties, aggregation, and fibrillation of human $\alpha$-synuclein. Biochemistry 2001, 40, 11604-11613. [CrossRef] [PubMed]

15. El-Agnaf, O.; Jakes, R.; Curran, M.D.; Wallace, A. Effects of the mutations Ala30 to Pro and Ala53 to Thr on the physical and morphological properties of $\alpha$-synuclein protein implicated in Parkinson's disease. FEBS Lett. 1998, 440, 67-70. [CrossRef]

16. Coskuner, O.; Wise-Scira, O. Structures and free energy landscapes of the A53T mutant-type $\alpha$-synuclein protein and impact of A53T mutation on the structures of the wild-type $\alpha$-synuclein protein with dynamics. ACS Chem. Neurosci. 2013, 4, 1101-1113. [CrossRef] [PubMed]

17. Sahay, S.; Ghosh, D.; Dwivedi, S.; Anoop, A.; Mohite, G.M.; Kombrabail, M.; Krishnamoorthy, G.; Maji, S.K. Familial Parkinson disease-associated mutations alter the site-specific microenvironment and dynamics of $\alpha$-synuclein. J. Biol. Chem. 2015, 290, 7804-7822. [CrossRef] [PubMed]

18. Tosatto, L.; Horrocks, M.H.; Dear, A.J.; Knowles, T.P.; Dalla Serra, M.; Cremades, N.; Dobson, C.M.; Klenerman, D. Single-molecule FRET studies on $\alpha$-synuclein oligomerization of Parkinson's disease genetically related mutants. Sci. Rep. 2015, 5, 16696. [CrossRef] [PubMed]

19. Chen, S.W.; Drakulic, S.; Deas, E.; Ouberai, M.; Aprile, F.A.; Arranz, R.; Ness, S.; Roodveldt, C.; Guilliams, T.; De-Genst, E.J. Structural characterization of toxic oligomers that are kinetically trapped during $\alpha$-synuclein fibril formation. Proc. Natl. Acad. Sci. USA 2015, 112, E1994-E2003. [CrossRef] [PubMed]

20. Stefanovic, A.N.; Lindhoud, S.; Semerdzhiev, S.A.; Claessens, M.M.; Subramaniam, V. Oligomers of Parkinson's disease-related $\alpha$-Synuclein mutants have similar structures but distinctive membrane permeabilization properties. Biochemistry 2015, 54, 3142-3150. [CrossRef] [PubMed]

21. Ferreon, A.C.M.; Gambin, Y.; Lemke, E.A.; Deniz, A.A. Interplay of $\alpha$-synuclein binding and conformational switching probed by single-molecule fluorescence. Proc. Natl. Acad. Sci. USA 2009, 106, 5645-5650. [CrossRef] [PubMed]

22. Sierecki, E.; Giles, N.; Bowden, Q.; Polinkovsky, M.E.; Steinbeck, J.; Arrioti, N.; Rahman, D.; Bhumkar, A.; Nicovich, P.R.; Ross, I. Nanomolar oligomerization and selective co-aggregation of $\alpha$-synuclein pathogenic mutants revealed by single-molecule fluorescence. Sci. Rep. 2016, 6, 37630. [CrossRef] [PubMed]

23. Uversky, V.N.; Li, J.; Souillac, P.; Millett, I.S.; Doniach, S.; Jakes, R.; Goedert, M.; Fink, A.L. Biophysical properties of the synucleins and their propensities to fibrillate inhibition of $\alpha$-synuclein assembly by $\beta$-and $\gamma$-synucleins. J. Biol. Chem. 2002, 277, 11970-11978. [CrossRef] [PubMed]

24. Jakes, R.; Spillantini, M.G.; Goedert, M. Identification of two distinct synucleins from human brain. FEBS Lett. 1994, 345, 27-32. [CrossRef]

25. Janowska, M.K.; Wu, K.-P.; Baum, J. Unveiling transient protein-protein interactions that modulate inhibition of $\alpha$-synuclein aggregation by $\beta$-synuclein, a pre-synaptic protein that co-localizes with $\alpha$-synuclein. Sci. Rep . 2015, 5. [CrossRef] [PubMed] 
26. Hashimoto, M.; Rockenstein, E.; Mante, M.; Mallory, M.; Masliah, E. $\beta$-Synuclein inhibits $\alpha$-synuclein aggregation: A possible role as an anti-parkinsonian factor. Neuron 2001, 32, 213-223. [CrossRef]

27. Park, J.-Y.; Lansbury, P.T. $\beta$-Synuclein inhibits formation of $\alpha$-synuclein protofibrils: A possible therapeutic strategy against Parkinson's Disease. Biochemistry 2003, 42, 3696-3700. [CrossRef] [PubMed]

28. Wright, J.A.; McHugh, P.C.; Pan, S.; Cunningham, A.; Brown, D.R. Counter-regulation of $\alpha$-and $\beta$-synuclein expression at the transcriptional level. Mol. Cell. Neurosci. 2013, 57, 33-41. [CrossRef] [PubMed]

29. Windisch, M.; Hutter-Paier, B.; Rockenstein, E.; Hashimoto, M.; Mallory, M.; Masliah, E. Development of a new treatment for Alzheimer's disease and Parkinson's disease using anti-aggregatory $\beta$-synuclein-derived peptides. J. Mol. Neurosci. 2002, 19, 63-69. [CrossRef] [PubMed]

30. Shaltiel-Karyo, R.; Frenkel-Pinter, M.; Egoz-Matia, N.; Frydman-Marom, A.; Shalev, D.E.; Segal, D.; Gazit, E. Inhibiting $\alpha$-synuclein oligomerization by stable cell-penetrating $\beta$-synuclein fragments recovers phenotype of Parkinson's disease model flies. PLoS ONE 2010, 5, e13863. [CrossRef] [PubMed]

31. Hashimoto, M.; Rockenstein, E.; Mante, M.; Crews, L.; Bar-On, P.; Gage, F.; Marr, R.; Masliah, E. An antiaggregation gene therapy strategy for Lewy body disease utilizing $\beta$-synuclein lentivirus in a transgenic model. Gene Ther. 2004, 11, 1713-1723. [CrossRef] [PubMed]

32. Tsigelny, I.F.; Bar-On, P.; Sharikov, Y.; Crews, L.; Hashimoto, M.; Miller, M.A.; Keller, S.H.; Platoshyn, O.; Yuan, J.X.J.; Masliah, E. Dynamics of $\alpha$-synuclein aggregation and inhibition of pore-like oligomer development by $\beta$-synuclein. FEBS J. 2007, 274, 1862-1877. [CrossRef] [PubMed]

33. Brown, J.W.; Buell, A.K.; Michaels, T.C.; Meisl, G.; Carozza, J.; Flagmeier, P.; Vendruscolo, M.; Knowles, T.P.; Dobson, C.M.; Galvagnion, C. $\beta$-Synuclein suppresses both the initiation and amplification steps of $\alpha$-synuclein aggregation via competitive binding to surfaces. Sci. Rep. 2016, 6. [CrossRef] [PubMed]

34. Fan, Y.; Limprasert, P.; Murray, I.V.; Smith, A.C.; Lee, V.M.-Y.; Trojanowski, J.Q.; Sopher, B.L.; La Spada, A.R. $\beta$-synuclein modulates $\alpha$-synuclein neurotoxicity by reducing $\alpha$-synuclein protein expression. Hum. Mol. Genet. 2006, 15, 3002-3011. [CrossRef] [PubMed]

35. Fujita, M.; Sugama, S.; Sekiyama, K.; Sekigawa, A.; Tsukui, T.; Nakai, M.; Waragai, M.; Takenouchi, T.; Takamatsu, Y.; Wei, J. A $\beta$-synuclein mutation linked to dementia produces neurodegeneration when expressed in mouse brain. Nat. Commun. 2010, 1, 110. [CrossRef] [PubMed]

36. Wei, J.; Fujita, M.; Nakai, M.; Waragai, M.; Watabe, K.; Akatsu, H.; Rockenstein, E.; Masliah, E.; Hashimoto, M. Enhanced lysosomal pathology caused by $\beta$-synuclein mutants linked to dementia with lewy bodies. J. Biol. Chem. 2007, 282, 28904-28914. [CrossRef] [PubMed]

37. Gambin, Y.; Polinkovsky, M.; Francois, B.; Giles, N.; Bhumkar, A.; Sierecki, E. Confocal spectroscopy to study dimerization, oligomerization and aggregation of proteins: A practical guide. Int. J. Mol. Sci. 2016, 17, 655. [CrossRef] [PubMed]

38. Kiely, A.P.; Ling, H.; Asi, Y.T.; Kara, E.; Proukakis, C.; Schapira, A.H.; Morris, H.R.; Roberts, H.C.; Lubbe, S.; Limousin, P. Distinct clinical and neuropathological features of G51D SNCA mutation cases compared with SNCA duplication and H50Q mutation. Mol. Neurodegener. 2015, 10, 41. [CrossRef] [PubMed]

39. Lesage, S.; Anheim, M.; Letournel, F.; Bousset, L.; Honoré, A.; Rozas, N.; Pieri, L.; Madiona, K.; Dürr, A.; Melki, R. G51D $\alpha$-synuclein mutation causes a novel Parkinsonian-pyramidal syndrome. Ann. Neurol. 2013, 73, 459-471. [CrossRef] [PubMed]

40. Snyder, H.; Mensah, K.; Hsu, C.; Hashimoto, M.; Surgucheva, I.G.; Festoff, B.; Surguchov, A.; Masliah, E.; Matouschek, A.; Wolozin, B. $\beta$-synuclein reduces proteasomal inhibition by $\alpha$-synuclein but not $\gamma$-synuclein. J. Biol. Chem. 2005, 280, 7562-7569. [CrossRef] [PubMed]

41. Beyer, K.; Domingo-Sàbat, M.; Santos, C.; Tolosa, E.; Ferrer, I.; Ariza, A. The decrease of $\beta$-synuclein in cortical brain areas defines a molecular subgroup of dementia with Lewy bodies. Brain 2010, 133, 3724-3733. [CrossRef] [PubMed]

42. Mureev, S.; Kovtun, O.; Nguyen, U.T.; Alexandrov, K. Species-independent translational leaders facilitate cell-free expression. Nat. Biotechnol. 2009, 27, 747-752. [CrossRef] [PubMed]

43. Kovtun, O.; Mureev, S.; Jung, W.; Kubala, M.H.; Johnston, W.; Alexandrov, K. Leishmania cell-free protein expression system. Methods 2011, 55, 58-64. [CrossRef] [PubMed]

44. Johnston, W.A.; Alexandrov, K. Production of eukaryotic cell-free lysate from Leishmania tarentolae. Methods Mol. Biol. 2014, 1118, 1-15. [PubMed] 
45. Gagoski, D.; Mureev, S.; Giles, N.; Johnston, W.; Dahmer-Heath, M.; Škalamera, D.; Gonda, T.J.; Alexandrov, K. Gateway-compatible vectors for high-throughput protein expression in pro-and eukaryotic cell-free systems. J. Biotechnol. 2015, 195, 1-7. [CrossRef] [PubMed]

46. Walhout, A.J.; Temple, G.F.; Brasch, M.A.; Hartley, J.L.; Lorson, M.A.; van den Heuvel, S.; Vidal, M. GATEWAY recombinational cloning: Application to the cloning of large numbers of open reading frames or ORFeomes. Methods Enzymol. 2000, 328, 575-592. [PubMed]

(c)

(C) 2018 by the authors. Licensee MDPI, Basel, Switzerland. This article is an open access article distributed under the terms and conditions of the Creative Commons Attribution (CC BY) license (http:/ / creativecommons.org/licenses/by/4.0/). 